\title{
Higher derivative discontinuous solutions to linear ordinary differential equations: A new route to complexity?
}

\author{
Dhurjati Prasad Datta*and Manoj Kumar Bose ${ }^{\dagger}$ \\ Department of Mathematics, North Bengal University, \\ P.O. North Bengal University, Darjeeling, India, Pin: 734430
}

\begin{abstract}
We present a new one parameter family of second derivative discontinuous solutions to the simplest scale invariant linear ordinary differential equation. We also point out how the construction could be extended to generate families of higher derivative discontinuous solutions as well. The discontinuity can occur only for a subset of even order derivatives, viz.,2nd, 4th, 8th, 16th, ....The solutions are shown to break the discrete parity (reflection) symmetry of the underlying equation. These results are expected to gain significance in the contemporary search of a new dynamical principle for understanding complex phenomena in Nature.
\end{abstract}

Chaos, Solitons and Fractals, vol 22, issue 2, (2004), 271-275

${ }^{*}$ Corresponding author, email:dp_datta@yahoo.com

$\dagger$ †email:manojkbose@rediffmail.com 


\section{Introduction}

Let us consider the simplest scale invariant linear ordinary differential equation (ODE)

$$
t \frac{\mathrm{d} \tau}{\mathrm{d} t}=\tau
$$

A well known fact of the standard analysis (calculus) is that this equation, and more generally, an ODE of the form $\frac{\mathrm{d} \tau}{\mathrm{d} t}=f(\tau, t)$ possesses a unique $C^{r}$ solution in a neighbourhood of $\left(t_{0}, \tau_{0}\right), \tau_{0}=\tau\left(t_{0}\right)$ when $f \in C^{r}$. This is the Picard's theorem. The standard solution of eq(1), as admitted by the Picard's theorem, is of course $\tau_{s}(t)=t$ when the initial condition is chosen as $\tau(1)=1$. Clearly, this is a $C^{\infty}$ solution. In the following section, we, however, present, in contradiction to the Picard's theorem, a new exact solution of eq(1), which is only $C^{1}$. The new solution breaks the reflection symmetry $(t \rightarrow-t)$ of the underlying ODE spontaneously. We interprete the solution in Sec. 3 and show how this defines a nonstandard extension of the real number system. Consequently, every real number is identified with an equivalence class of a continuum of new, infinitesimally separated elements, which are in a state of random fluctuations. We also point out how the new construction generalizes to create higher derivative $(4 \mathrm{th}, 8 \mathrm{th}, \ldots)$ discontinuous families of solutions to the above equation. The results presented here are new and sums up our earlier investigations [1, 2] of eq(11) in a mathematically rigorous manner. These rigorous results should become important in the contemporary search of a new dynamical principle for understanding complexity at different levels of life viz., physical, biological, financial and social [3, 4, 5], besides their deep significance in mathematical analysis. Ref [2] contains some preliminary results and definitions indicating their role in defining a new framework for addressing living systems (see also [6] ).

\section{$2 \quad C^{1}$ solution}

Let us first demonstrate the existence of such a solution in the neighbourhood of $t=1$. The solution would only have a continuous first order derivative at $t=1$. We begin by defining our notations.

Let $t_{n \pm}=1 \pm \eta_{n}, t_{0} \equiv t, 0<\eta_{n}<<1, \alpha_{n}=1+\epsilon_{n}, n=0,1,2, \ldots$, and $\epsilon_{0}=0,0<\epsilon_{n}<1(n \neq 0)$, such that $\epsilon_{n} \rightarrow 0$, as $n \rightarrow \infty$ (we retain the symbol $\alpha_{0}$ for the sake of symmetry). Next, we write $t_{n \pm}^{\prime}=1 \pm \alpha_{n} \eta_{n}^{\prime}$, so that $\alpha_{n} t_{n-}=t_{n-}^{\prime}$. Consequently, $\eta_{n}^{\prime}=\eta_{n}-\frac{\epsilon_{n}}{\alpha_{n}}$. Here, $\alpha_{n}$ (and $\left.\epsilon_{n}\right)$ are scaling parameters. A useful choice, however, is $\epsilon_{n}=\epsilon^{2^{n}}, \epsilon=\epsilon_{1}$ (the reason will become clear later). As will become evident, $\eta_{n+1}=\alpha_{n}^{2} \eta_{n}^{\prime 2}$.

To construct a nontrivial solution (with the initial condition $\tau(1)=1$ ), we begin with an initial approximate solution in the small scale variable $\eta_{0}$, and then obtain recursively self-similar corrections over smaller and smaller scale variables $\eta_{0}^{2}, \eta_{0}^{4}, \ldots$ To this end, let

$$
\tau(t)=\left\{\begin{array}{ll}
\tau_{-} & \text {if } t \lesssim 1 \\
\tau_{+} & \text {if } t \geqq 1
\end{array}, \tau_{-}\left(t_{-}\right)=\left(1 / t_{+}^{\prime}\right) f_{1-}\left(\eta_{0}\right), \tau_{+}\left(t_{+}\right)=t_{+}\right.
$$

be an exact solution of eq(1). This is obviously true for the right hand component $\tau_{+}$. To verify the same for the nontrivial component $\tau_{-}$, we differentiate it with respect to $t_{-}$, and use the scale invariance of eq(1). Utilizing $\alpha_{0} t_{-}=t_{-}^{\prime}$, one obtains

$$
t_{-}^{\prime} \frac{\mathrm{d} \tau_{-}}{\mathrm{d} t_{-}^{\prime}}=\tau_{-}\left(\frac{t_{-}^{\prime}}{t_{+}^{\prime}}-t_{-}^{\prime} \frac{f_{1-}^{\prime}}{f_{1-}}\right)
$$

where $f_{1-}^{\prime}=\frac{\mathrm{d} f_{1-}}{\mathrm{d} \bar{\eta}_{0}}, \bar{\eta}_{0}=\alpha_{0} \eta_{0}^{\prime}$. Consequently, eq(2) would be an exact solution if and only if $f_{1-}$ solves exactly the self-similar equation

$$
t_{1-} \frac{\mathrm{d} f_{1-}}{\mathrm{d} t_{1-}}=f_{1-}
$$

in the smaller logarithmic variable $\ln t_{1-}^{-1}$, where $t_{1-}=1-\alpha_{0}^{2} \eta_{0}^{\prime 2} \equiv 1-\eta_{1}$. The self-similar replica eq(41) follows from the equality 


$$
\frac{t_{-}^{\prime}}{t_{+}^{\prime}}-t_{-}^{\prime} \frac{f_{1-}^{\prime}}{f_{1-}}=1
$$

so that $\tau_{-}$is an exact solution of eq(11). The exact (nontrivial part of the ) solution could thus be written recursively as

$$
\tau_{-}=C \frac{1}{t_{+}} \frac{1}{t_{1+}^{\prime}} \cdots \frac{1}{t_{(n-1)+}^{\prime}} f_{n-}\left(\eta_{n}^{\prime}\right)
$$

where $f_{n}$ satisfies the $n$th generation self-similar equation

$$
t_{n-} \frac{\mathrm{d} f_{n-}}{\mathrm{d} t_{n-}}=f_{n-}
$$

and $t_{n-}=1-\alpha_{n-1}^{2} \eta_{n-1}^{\prime 2} \equiv 1-\eta_{n}$. We also note that $t_{+}^{\prime}=t_{+}$, since $\alpha_{0}=1$.

Plugging in the initial condition $\tau_{ \pm}=1$ at $t_{ \pm}=1$ (viz., $\eta_{0}=0$ ), one obtains finally the desired solution as

$$
\tau_{-}=C \frac{1}{t_{+}} \frac{1}{t_{1+}^{\prime}} \frac{1}{t_{2+}^{\prime}} \ldots, \tau_{+}=t_{+}
$$

where $C=t_{1+}^{\prime}(0) t_{2+}^{\prime}(0) \ldots$ Notice that $C \neq 1$, since $\eta_{1}^{\prime}=-\epsilon_{1} / \alpha_{1}, \eta_{2}^{\prime}=\epsilon_{1}^{2}-\epsilon_{2} / \alpha_{2}$, etc, when $\eta_{0}=0$.

A remark is in order here.

The solution eq(8) follows from eq(6) only if the sequence $\left\{f_{n-}\right\}$ is convergent. In fact, we prove that $f_{\infty}=\lim _{n \rightarrow \infty} f_{n-}=1$. Let $\tau_{n}=\frac{1}{t_{+}} \frac{1}{t_{1+}^{\prime}} \ldots \frac{1}{t_{n+}^{\prime}}$. Then for $\eta_{0}$ sufficiently small and $\epsilon_{n} \rightarrow 0$ for $n \rightarrow \infty$, the sequence $\left\{\tau_{n}\right\}$ is convergent (to a nonzero value), since $t_{n+}^{\prime} \rightarrow 1$ as $n \rightarrow \infty$. Accordingly, for $\varepsilon>0, \exists N_{1}$ such that $\left|\tau_{m}-\tau_{n}\right|<\varepsilon$ for $m, n>N_{1}(m>n)$. As a result, $0<k_{1}<\tau_{n}<k_{2}, k_{1}, k_{2} \sim$ $\mathrm{O}(1)$, for $n>N_{2}$ for a sufficiently large $N_{2}$. Again, $f_{n-}$, being defined by eq(7), is uniformly bounded in a neighbourhood of $t=1$, so that $\left|f_{n-}\right|<k$ for $n>N_{2}$. The desired convergence now follows from the Cauchy convergence criterion, since $\left|f_{n-}-f_{m-}\right|=\left|\tau_{n}^{-1}\right|\left|f_{m-}\right|\left|\tau_{m}-\tau_{n}\right|<k_{1}^{-1} k \varepsilon \forall m, n>N, N=$ $\max \left(N_{1}, N_{2}\right)$. Finally, eq(7), in the asymptotic limit $n \rightarrow \infty$, yields $f_{\infty}=\left.\frac{\mathrm{d} f_{n-}}{\mathrm{d} t_{n-}}\right|_{\infty}=\left.\frac{\mathrm{d} \tau}{\mathrm{d} t}\right|_{t=1}=\tau(1)=1$.

Now to test the continuity of the derivatives of the solution (8) at $t_{ \pm}=1$, i.e., at $\eta_{0}=0$, we note that $\eta_{n}^{\prime}$ is a polynomial in $\eta_{0}$, of degree $2^{n}$, being defined recursively by $\eta_{n}^{\prime}=\eta_{n}-\frac{\epsilon_{n}}{\alpha_{n}}, \eta_{n}=\alpha_{n-1}^{2} \eta_{n-1}^{\prime 2}$. As a result $\frac{\mathrm{d} \eta_{n}^{\prime}}{\mathrm{d} \eta_{0}}=0$, but $\frac{\mathrm{d}^{2} \eta_{n}^{\prime}}{\mathrm{d} \eta_{0}^{2}} \neq 0$, at $\eta_{0}=0$. One thus obtains

$$
\frac{\mathrm{d} \tau_{-}}{\mathrm{d} \eta_{0}}=-\tau_{-}\left\{\frac{1}{1+\eta_{0}}+\left(\frac{\alpha_{1}}{1+\alpha_{1} \eta_{1}^{\prime}}\right) \frac{\mathrm{d} \eta_{1}^{\prime}}{\mathrm{d} \eta_{0}}+\left(\frac{\alpha_{2}}{1+\alpha_{2} \eta_{2}^{\prime}}\right) \frac{\mathrm{d} \eta_{2}^{\prime}}{\mathrm{d} \eta_{0}}+\ldots\right\}
$$

so that $\frac{\mathrm{d} \tau_{-}}{\mathrm{d} t_{-}}=1=\frac{\mathrm{d} \tau_{+}}{\mathrm{d} t_{+}}$at $\eta_{0}=0$ which means that the first derivative of the solution is indeed continuous for all $\eta_{0}$. However, as is verified easily from eq(9), the second derivative of $\tau_{-}$at $\eta_{0}=0$ is not zero, as one expects on the basis of the standard solution $\tau_{s}=t$. Indeed, one can verify that $\frac{\mathrm{d}^{2} \tau_{-}}{\mathrm{d} \eta_{0}^{2}}=2\left(1-\frac{1+\epsilon_{1}}{1-\epsilon_{1}}-\ldots\right) \neq 0$ at $\eta_{0}=0$, unless $\epsilon_{n}=0$, for all $n$. In this special case, i.e., when $\epsilon_{n}=0, \forall n$, our solution (86) reduces to the standard solution, since $\tau_{-}=\frac{1}{1+\eta_{0}} \frac{1}{1+\eta_{0}^{2}} \frac{1}{1+\eta_{0}^{4}} \ldots=1-\eta_{0}=t_{-}$.

It thus follows that the solution (8), with nonzero scaling parameters, is indeed nontrivial, because of this second derivative discontinuity at $\eta_{0}=0$, that is at $t=1$. In fact, the scaling invariance of eq(11) tells also that, $t=1$ could be realized as $t \rightarrow t / t_{0}=1$, so that the nontrivial solution (8) actually holds in the neighbourhood of every real number $t_{0}$, the 2 nd derivative being discontinuous at $t=t_{0}$. This is our main result of this paper. Let us note here that $\tau_{-}=\tau_{s-}\left(1+\mathrm{O}\left(\eta_{0}^{2}\right)\right)$, besides the arbitrariness of the scaling parameters $\epsilon_{n}$. Combining the standard and the new solutions together, one can finally write down a more general one parameter class of solutions

$$
\tau_{g}(t)=t(1+\phi(t)), \phi(t)=\epsilon t^{-1} \tau(t)
$$

Note that 


$$
t \frac{\mathrm{d} \phi}{\mathrm{d} t}=0
$$

because $\tau$ is an exact solution of eq(1) (we fix $\epsilon_{n}=\epsilon^{2^{n}}$, on anticipation of Sec.3). The 2nd derivative discontinuity of $\tau$, however, tells that $\phi$ can not be considered simply as an ordinary constant [1, 2].

\subsection{Symmetry breaking}

The solution (8) is not parity (reflection) symmetric. Let $P: P t_{ \pm}=t_{\mp}$ denote the reflection transformation near $t=1(P \eta=-\eta$ near $\eta=0)$. Clearly, eq(1) is parity symmetric. So is the standard solution $\tau_{s \pm}=t_{ \pm}\left(\right.$since $\left.P \tau_{s}=\tau_{s}\right)$. However, the solution (8) breaks this discrete symmetry spontaneously: $\tau_{-}^{P}=P \tau_{+}=t_{-}, \tau_{+}^{P}=P \tau_{-}=C \frac{1}{t_{-}} \frac{1}{t_{1+}^{\prime}} \frac{1}{t_{2+}^{\prime}} \ldots$, which is of course a solution of eq(1), but clearly differs from the original solution, $\tau_{ \pm}^{P} \neq \tau_{ \pm}$. The explicit breaking of the parity symmetry is an important new feature of the solution (8). Its significance in dynamical systems would be studied elsewhere.

\section{Interpretation}

A basic assumption in the framework of the standard calculus is that a real variable $t$ changes by linear translation only. Further, $t$ assumes (attains) every real number exactly. However, in every computational problem within a well specified error bar, a real number is determined only upto a finite degree of accuracy $\epsilon_{0}$,say. Suppose, for example, in a computation, 1 is determined upto an accuracy of \pm 0.01 , so that 1 here effectively stands for the set $1_{\epsilon} \equiv\left\{1 \pm \epsilon, \epsilon<\epsilon_{0}=0.01\right\}$ with cardinality $c$, the cardinality of the continuum. We call $\epsilon$ an 'infinitesimally small' real number. Now, any laboratory computational problem (experiment) is run only over a finite time span, and the influence of such infinitesimally small $\epsilon$ 's, being insignificantly small, could in fact be disregarded. However, if the experiment is allowed to run over an 'infinitely' large period of time, analogous to a natural (or biological/ financial) process, such as the fluctuations of a river (ocean) water level (heart beats of a mammal/ stock market prices ), which is known to occur over many time scales, the contributions from the infinitesimally small scales $\epsilon$ need not remain negligible.

Our solution (8) captures the essence of the above scenario in an elegant manner. Notice that our initial ansatz $\tau_{-} \approx 1 / t_{+}$, approximates the standard solution $\tau_{s-}=t_{-}$upto $\mathrm{O}\left(\eta_{0}^{2}\right)$ (i.e., when $\eta_{0} \neq 0$, but $\left(\mathrm{O}\left(\eta_{0}^{2}\right)=0\right)$. At this level of approximation $1_{\epsilon}=1$ for $\epsilon \lesssim \eta^{2}$. However, at the second level of iteration with the variable $t_{1-}=1-\eta_{0}^{2}$, the effect of $\epsilon$, being of the same order of $\eta_{0}^{2}$, can not be negligible. Recalling that 1 here should be identified as $1 \pm \epsilon$, an appropriate variable can therefore be written as $\alpha t_{1-}=1 \pm \epsilon-\alpha \eta_{0}^{2}$, which in turn is written equivalently as the rescaled variable $t_{1-}^{\prime}=1-\alpha \eta_{1}^{\prime}$ where $\eta_{1}^{\prime}=\eta_{0}^{2} \mp \epsilon / \alpha$ so that $t_{1-}^{\prime}=\alpha t_{1-}, \alpha=1 \pm \epsilon$. This scaling transformation, which is used recursively in (8), thus not only reveals an intrinsically approximate nature of $1_{\epsilon}$, but also provides an window to probe the fine scale structure of $1_{\epsilon}$ by recursively approaching more and more accurately the 'exact' value 1 through the finer and finer scales $\epsilon^{2}, \epsilon^{4}, \ldots$ successively. ( This explains the choice $\epsilon_{n}=\epsilon^{2^{n}}$.) The exact value of 1 (and, hence, of any real number), as endowed in the framework of standard classical analysis, is, however, illusory, because of the fact that the solution (8), being 2nd derivative discontinuous at $t=1$, is a new exact solution of eq(11). Further, the value of a small real number $\eta_{0}$ is uncertain not only upto $\mathrm{O}\left(\eta_{0}^{2}\right)$, but also because of the arbitrary parameter $\epsilon$. We note that two solutions $\tau_{g}$ and $\tau_{s}$ are indistinguishable for $t \sim \mathrm{O}(1)$ and $\eta_{0}^{2}<<1$. However, for a sufficiently large $t \sim \mathrm{O}\left(\epsilon^{-1}\right)\left(\equiv \mathrm{O}\left(\eta_{0}^{-2}\right)\right)$, the behaviours of two solutions would clearly be different. Moreover, because of the intrinsic (irreducible) uncertainty the new solution $\tau_{g}$ could effectively be written as $\tau_{g}: \tau_{-} \approx 1 / t_{+}, \tau_{+}=t_{+}$, without any loss of generality (and information) at the order $\eta_{0} \neq 0, \eta_{0}^{2}=0$. The correction factors in eq(8) only reveals the approximate (statistical) self similarity of the solution over higher order scales $\eta_{0}^{2}, \eta_{0}^{4}, \ldots$ and so on.

To summarise, the nature of the solution (8) depends crucially on the nontrivial scaling $\alpha_{n} t_{-}=t_{-}^{\prime}$, as defined above, which shifts $\eta_{0}=0$ at the level of first iteration to $\eta_{1}^{\prime}=0$ for the second iteration 
and so on recursively to finer and finer scales. The origin of the second derivative discontinuity is obviously an effect of this receding of the zero (0) down finer scales. Let us also point out that the scaling $\alpha t_{-}=t_{-}^{\prime}$, does not mean $\alpha t_{+}=t_{+}^{\prime}$ (in fact, $t_{+}^{\prime}=(1-\epsilon)\left(1+\frac{1+\epsilon}{1-\epsilon} \eta_{0}\right) \neq \alpha t_{+}$), which in turn leads to the nontrivial feature that is revealed in the solution (8).

Now to state the above more formally, we note that the standard solution $\tau_{s}=t$ defines the 1-1 identity map $R \rightarrow R$ of the real number set $R$. The generalized solution (10) now provides a one parameter extension of the identity map $\mathbf{1}_{\epsilon}: R \rightarrow R$. Accordingly, every real number $t$ is mapped to the corresponding 'fat' real number $\mathbf{t}_{\epsilon}=t(1+\phi)$, which is the set of 'infinitely many' new elements (with cardinality $2^{c}$ ) separated by infinitesimally small numbers (distances) [1], since $\phi$ is infinitesimally small. Accordingly, the set of real numbers $R$ is considered to be an enlarged nonstandard set [7] $\mathbf{R}$ with infinitesimally small elements, which remain dormant in the standard analysis (i.e., in the context of the standard solution $\tau_{s}$ of eq(1) $)$. This extension of $R$ to the nonstandard set $\mathbf{R}$ via the generalized solution of the linear ODE eq(1), is analogous to the extension of the ring of integers to the field of rational numbers as the solution space of the linear equation $a x+b=0, a, b$ being integers. Let us note further that eq(9) written as

$$
\mathrm{d} \tau_{-}=-\tau_{-}\left\{\frac{\mathrm{d} \eta_{0}}{1+\eta_{0}}+\frac{\alpha_{1} \mathrm{~d} \eta_{1}^{\prime}}{1+\alpha_{1} \eta_{1}^{\prime}}+\frac{\alpha_{2} \mathrm{~d} \eta_{2}^{\prime}}{1+\alpha_{2} \eta_{2}^{\prime}}+\ldots\right\}
$$

tells that the variables $t_{+}, t_{1+}^{\prime}, \ldots$ behave as independent variables. Writing $\mathbf{t}_{-}^{-1}=\Pi_{0}^{\infty} t_{n+}^{\prime}$, a fat real variable in the neighbourhood of $\mathbf{1}_{\epsilon}$, eq(12) restates the fact that the solution eq(8) is indeed an 'exact'

solution of the equation $\frac{\mathrm{d} \tau}{\mathrm{d} \ln \mathbf{t}_{-}}=\tau$ in $\mathbf{R}$. In $R$, however, we have an irreducible 'unknown' component $f_{n-} \sim \mathrm{O}(1)$, which is exactly determined only in the limit $n \rightarrow \infty$. Ironically, however, even in this asymptotic limit, one fails to improve (determine) the solution with an unlimited accuracy, because of the indeterminate parameter $\epsilon$, as reflected in the 2 nd derivative discontinuity. The fat variable reduces to the ordinary real variable $t_{-}$only in the approximation when $\eta_{0}^{2}=0\left(\eta_{0} \neq 0\right)$.

The solution (8) also tells that a fat real variable $\mathbf{t}=t \mathbf{1}$ in $\mathbf{R}$ can change not only by linear translations but also by inversions: $t_{-} \rightarrow t_{-}^{-1}=t_{+}$, in the neighbourhood of $t=1$. Clearly, the inversion is realized 'exactly' in $\mathbf{R}$, when an (first order) infinitesimal $\phi$ is defined by $\phi \neq 0$, but $\mathrm{O}\left(\phi^{2}\right)=0$. Consequently, the change (increment) of a real variable could be visualized as an $\mathrm{SL}(2, \mathrm{R})$ group action, viz., a combination of linear translation ( for ordinary real variable $t$ following the standard solution of eq(10) and inversion ( for $t_{ \pm} \in \mathbf{1}$ ). As note already, because of the arbitrariness in $\epsilon$, as well as being defined upto $\mathrm{O}\left(\phi^{2}=0\right)$, the infinitesimal elements $\phi$ enjoy an element of randomness, which in turn renders $\mathbf{t}$ random. The ordinary non-random real variable $t$ is thus retrieved only under an approximation, that is, when $\phi=0$.

Let us remark finally that one can generate 4th or higher derivative discontinuous solutions of eq(11) by introducing infinitesimal scalings at an appropriate level of iteration. Note that the 2nd derivative discontinuous solution is obtained when the nontrivial scaling viz., $t_{1-} \rightarrow t_{1-}^{\prime}$ is introduced at the 1st iteration (the ansatz in eq(2) stands for the zeroth iteration ). Thus, the 4th derivative discontinuous solution is obtained when the rescaling of the appropriate variable is used at the 2 nd level of the iteration, viz., $t_{2-} \rightarrow t_{2-}^{\prime}$, giving rise to yet another class of solutions

$$
\tau_{-}^{(2)}=C \frac{1}{t_{+}} \frac{1}{t_{1+}} \frac{1}{t_{2+}^{\prime}} \% \operatorname{ldots} \frac{1}{t_{(n-1)+}^{\prime}} f_{n-}\left(\eta_{n}^{\prime}\right)
$$

where $t_{1+}=1+\eta_{0}^{2}, t_{2+}^{\prime}=1-\epsilon+(1+\epsilon) \eta_{0}^{4}, t_{3+}^{\prime}=1-\epsilon^{2}+\left(1+\epsilon^{2}\right)\left[(1+\epsilon) \eta_{0}^{4}-\epsilon\right]^{2}, \ldots$, and so on for higher derivative discontinuous solutions. Note that $\tau_{-}^{(2)}=\tau_{s}\left(1+\mathrm{O}\left(\eta_{0}^{4}\right)\right)$ for an $0 \neq \epsilon \lesssim \mathrm{O}\left(\eta_{0}^{4}\right)$. Consequently, $\ln \left(\tau_{-}^{(n)} / \tau_{s}\right) \approx \eta_{0}^{2^{n}}$ corresponds to the $n$th order infinitesimals in $\mathbf{R}$.

\section{Conclusion}

We have presented new families of higher derivative discontinuous solutions of the ODE (1), which do not respect the Picard's theorem. The origin of this violation could be traced to the fact that a 
variable in $\mathbf{R}$ may undergo changes (increments) via the extended $\mathrm{SL}(2, \mathrm{R})$-like group actions. These solutions break explicitly the parity symmetry of the underlying ODE. The derivation of such solutions from any linear first order ODE is obvious. Higher order equations will be considered elsewhere. We close with the observation that the results presented here may as well be considered to offer, so to speak, an exact proof of the fact that mathematics is inexact.

\section{References}

[1] D P Datta, A new class of scale free solutions to linear ordinary differential equations and the universality of the Golden Mean $\frac{\sqrt{5}-1}{2}=0.618033 \ldots$.. Chaos, Solitons \& Fractals, 17, (2003), 621-630.

[2] D. P. Datta, Dynamical real numbers and living systems, Chaos, Solitons 86 Fractals, 20,(2004), 705-712.

[3] I. Prigogine and I. Stenger, Order out of chaos: man's new dialogoue with nature, Bantum Books, New York (1984).

[4] P. Grigolini, P. Hamilton, J. Roberts and B. J. West, Towards a new vision of complexity, Chaos, Solitons $\&$ Fractals, 20, (2004), 1-185 (special issue).

[5] M. S. El Nashie, A review of E infinity theory and the mass spectrum of high energy particle physics, Chaos, Solitons $\&$ Fractals, 19 (2004), 209-236.

[6] H. Weiss and V. Weiss, The golden mean as clock cycle of brain waves, Chaos, Solitons E Fractals, 18, 2003, 643-652.

[7] A Robinson, Nonstandard analysis, North-Holland, Amsterdam, (1966). 